\title{
Multi-Feature Fusion Based Mechanical Fault Diagnosis for On-Load Tap Changers in Smart Grid with Electric Vehicles
}

\author{
Yan Yan, Hongzhong Ma, Member, IEEE, Miaowen Wen, Senior Member, IEEE, \\ Shuping Dang, Member, IEEE, and Honghua Xu
}

\begin{abstract}
With the proliferation of electric vehicles (EVs), the supporting facilities and infrastructure become new components in conjunction with conventional electrical appliances. These novel appliances, e.g., charging piles and energy storage devices, bring new features as well as challenges to the existing power grid. To enhance the accuracy of mechanical fault identification for on-load tap changers (OLTCs) in smart grid with EVs, a feature selection method for OLTC mechanical fault identification is proposed in this paper. This method relies on the multi-feature fusion and the joint application of the K-nearest neighbors algorithm (KNN) and the improved whale optimization algorithm (IWOA). By multi-feature fusion, the high-dimensional set of time-domain and frequencydomain characteristics as well as energy and composite multi-scale permutation entropy can be constructed. As a result, the maximum correlation minimum redundancy (mRMR) principle can be used to screen the sensitive feature subsets. Finally, IWOA is used to optimize the sensitive feature subsets, and KNN is used to classify the different types of optimal feature subsets. The experimental results show that the proposed method is at least $8 \%$ more accurate than the existing methods. The high-accuracy nature of the proposed method can accelerate the promotion of EVs and the establishment of intelligent transportation environments.

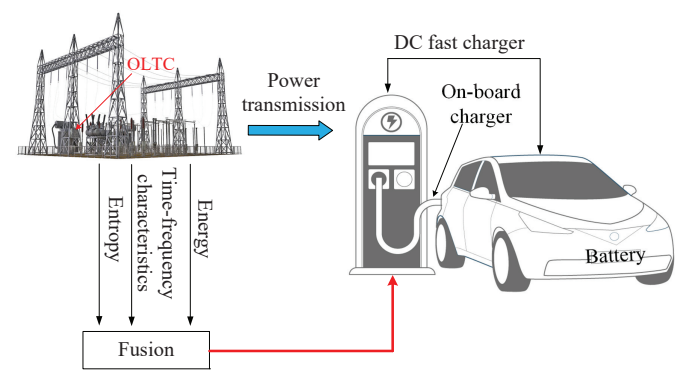

Index Terms-Mechanical fault diagnosis, multi-feature fusion, improved whale optimization algorithm (IWOA), on-load tap changer, electric vehicle (EV).

\section{INTRODUCTION}

$\mathbf{O}$ N-LOAD tap changers (OLTCs) play an important role in regulating reactive power and stabilizing grid voltage in power systems [1]. According to statistical reports, OLTCinduced transformer faults account for more than $20 \%$ of the total faults, and mechanical faults account for more than $90 \%$ of the total [2].In recent years, with the rapid application of electric vehicles (EVs), the matching charging piles and energy storage devices have been incorporated into the power grid [3]. These components inevitably bring new features and challenges to the traditional power grid. Specifically, a

This work was supported in part by the National Natural Science Foundation of China under Grant 51577050 and in part by the Fundamental Research Funds for the Central Universities under Grant 2019SJ02.

Y. Yan and H. Ma are with College of Energy and Electrical Engineering, Hohai University, Nanjing 211100, China (e-mail: $\{170206030008$, ma\}@hhu.edu.cn).

M. Wen is with the School of Electronic and Information Engineering, South China University of Technology, Guangzhou 510641, China (email: eemwwen@scut.edu.cn).

S. Dang is with Computer, Electrical and Mathematical Science and Engineering Division, King Abdullah University of Science and Technology (KAUST), Thuwal 23955-6900, Saudi Arabia (e-mail: shuping.dang@kaust.edu.sa).

H. Xu is with State Grid Jiangsu Electric Power Co., Ltd., Nanjing 320105, China (e-mail: honghuawl@126.com). 
the OLTC switching process in a non-interferential manner and obtains the state information and working condition of the OLTC switching process. The obtained information can be utilized to evaluate the operational status of OLTCs and diagnose faults. In this analytical method, how to identify the effective characteristic quantities from the collected vibration signals and combine them with an effective diagnosis method is the key. In [8]-[10], the time-domain characteristics of the OLTC vibration signals are determined by continuous wavelet. Accordingly, the working state criterion of the OLTC is established according to the ridge distribution diagram of the vibration signal. As a result, the characteristics of the ridge distribution diagrams regarding the typical faults of OLTCs are analyzed, and a mechanical fault diagnosis method of OLTC based on Kohonen's self-organizing mapping network is proposed.

For different stages of OLTC's switching process, Seo et al. proposed to use wavelet analysis to take the time and amplitude information of the OLTC vibration signal pulse as the characteristic parameters [11]. In this way, the abnormal pulse and action time sequence are associated with analyzing the operational status and identifying the abnormal states of specific parts. Due to the randomness and low-frequency chaos of the vibration signal in the OLTC switching process, the authors in [12], [13] propose an OLTC fault diagnosis model based on the power spectrum of the vibration signal and the hidden Markov chain. The proposed diagnosis model is capable of reconstructing the phase space of the OLTC vibration signal and identifying the working condition of the OLTC by defining the phase space coefficient. However, due to the limitations of the phase space reconstruction algorithm, the chaotic information of the signal cannot be fully retrieved.

In [1], the narrow-band noise assisted multi-variate empirical mode decomposition (EMD) algorithm is introduced into the mechanical fault monitoring for converters and OLTCs. The EMD algorithm mainly relies on the narrow-band noise signal to suppress the modal aliasing phenomenon and overcome some defects of the traditional methods. Experimental results have verified the high diagnostic accuracy of this method. However, the judging criterion for the characteristic matrix in this method is oversimple and needs further improvement.

In order to further improve the diagnostic accuracy of OLTC mechanical faults, we propose a mechanical fault feature selection method for OLTCs based on multi-feature fusion and IWOA-KNN. The main contributions of this paper are summarized as follows:

- By the quadratic penalty factor and the preset scale on the decomposition of the variable mode decomposition (VMD), the envelope entropy is chosen as the fitness function, and particle swarm optimization (PSO) is used to make the self-adaptive selection of its parameters.

- To overcome the defect of single feature selection and enhance diagnostic accuracy of OLTC mechanical faults, a high-dimensional collection of time-domain and frequency-domain characteristics as well as energy and composite multi-scale permutation entropy is constructed by multi-feature fusion.
- To reduce the redundancy and complexity brought by multi-feature fusion, the mRMR principle is used to screen the initial multi-feature fusion, and the diagnostic accuracy is chosen as the fitness function. In this way, IWOA is used to optimize the best feature subsets, and KNN is used to classify different types of the best feature subsets.

In addition, the high-accuracy nature of the proposed method is able to accelerate the promotion of EVs and the establishment of intelligent transportation environments. With the rapid developments of intelligent algorithms, information and communications technology (ICT) for the automobile industry [14][18], and with the applications of 5G wireless technologies for traffic control and optimization [19]-[21], it is expected that there will be a large number of intelligent EVs and electrical appliances that can benefit from the proposed mechanical fault diagnosis method. and with the application of $5 \mathrm{G}$ network in traffic ,it is expected that there will be a large number of intelligent EVs and electrical appliances that can benefit from the proposed mechanical fault diagnosis method.

The rest of this paper is organized as follows. In Section II, we introduce the multi-feature fusion from three perspectives. Then, we propose the feature selection model for OLTC mechanical faults in Section III. The experimental verification and discussion of the obtained results are presented in Section IV. Finally, we conclude the paper in Section V.

\section{Multi-Feature Fusion}

In this section, we introduce how multiple features can be fused to form the feature matrix that helps the diagnosis of mechanical faults in OLTCs. These features include energy characteristics, entropy characteristics, and time-frequency characteristics.

\section{A. Energy Characteristics and Entropy Characteristics}

VMD is a novel adaptive time-frequency analytical method based on Wiener filter, Hilbert transform, analytic signal, and heterodyne demodulation [22]-[24]. The essence of this decomposition is an iterative solution process for a specific model. By utilizing the VMD algorithm, the number of intrinsic mode function (IMF) components $K$ should be set in advance. For different $K$, the final signal processing result will be different, and the penalty parameter $\Psi$ has a great influence on the broadband of the modal components. This paper proposes to optimize the two parameters of the VMD algorithm by the PSO algorithm [25]. Following the paradigm given in [26], the envelope entropy of a signal $x(j)$ with length $N$ is adopted as the fitness function, and the definition of envelope entropy is as follows:

$$
E_{p}=-\sum_{j=1}^{N} p_{j} \log \left(p_{j}\right)
$$

where $a(j)$ is the envelope signal extracted from $x(j)$ after Hilbert demodulation; $p_{j}=\frac{a(j)}{\sum_{j=1}^{N} a(j)}$ is the normalized form of $a(j)$. In this paper, the minimum value of envelope entropy is taken as the fitness function value in the process 
of optimization. Therefore, the optimization on the fitness function is constructed as follows:

$$
\text { Minimize } E_{p} \text {. }
$$

Compared with a typical OLTC vibration signal, the energy of the OLTC fault vibration signal in the same frequency band is quite different, which contains rich fault information in the energy corresponding to each frequency component. Therefore, the IMF signal components decomposed by PSOVMD can be used as the characteristics to effectively extract most kinds of fault features of OLTC. The specific computing steps of energy characteristics are detailed as follows:

1) Decomposite the OLTC vibration signal $x(j)$ by PSOVMD and select $k$ IMF components;

2) Calculate the total energy of each signal's inherent modal function component by $E_{i}=\int_{-\infty}^{\infty}\left|c_{i}(t)\right|^{2} \mathrm{~d} t$, where $c_{i}(t)$ represents the magnitude of each scale coefficient or natural mode function at time $t$;

3) Calculate the quadratic sum of energy by $E_{\Sigma}=$ $\sqrt{\sum_{i=1}^{k}\left|E_{i}\right|^{2}}$

4) Construct feature vector $\mathbf{T}$ with feature energy as elements by $\mathbf{T}=\left[E_{k, 1}, E_{k, 2}, \ldots, E_{k, i}\right]$, where $E_{k, i}$ represents the $i$ th energy eigenvalue corresponding to the $k$ th IMF component;

5) Normalize $\mathbf{T}$ to yield the normalized feature vector $\mathbf{T}^{\prime}=$ $\left[E_{k, 1} / E_{\Sigma}, E_{k, 2} / E_{\Sigma}, \ldots, E_{k, i} / E_{\Sigma}\right]$.

\section{B. Composite Multi-Scale Permutation Entropy Characteristics}

The composite multi-scale permutation entropy (CMPE) algorithm optimizes the single coarsening in the classic MPE algorithm by means of composite coarsening. Through obtaining multiple composite coarsening time series on multiple scales, we can determine multiple permutation entropy values for multiple coarsening sequences. In this way, we can obtain the CMPE values by averaging multiple permutation entropy values [27]. After decomposing the OLTC vibration signal with the PSO-VMD algorithm, $k$ IMF are obtained, CMPE is calculated for each IMF component, and the CMPE matrix can be written as

$$
\mathbf{C M P E}=\left[\begin{array}{cccc}
\mathrm{CMPE}_{1,1} & \mathrm{CMPE}_{1,2} & \ldots & \mathrm{CMPE}_{1, s} \\
\mathrm{CMPE}_{2,1} & \mathrm{CMPE}_{2,2} & \ldots & \mathrm{CMPE}_{2, s} \\
\vdots & \vdots & \ddots & \vdots \\
\mathrm{CMPE}_{k, 1} & \mathrm{CMPE}_{k, 2} & \ldots & \mathrm{CMPE}_{k, s}
\end{array}\right] \text {, }
$$

where $\mathrm{CMPE}_{\nu, v}$ represents the CMPE value with scale $v$ of the $\nu$ th IMF component, $\forall \nu \in\{1,2, \ldots, k\}$ and $v \in$ $\{1,2, \ldots, s\}$. With CMPE, we can average each column of elements 1 to $k$ in the matrix one by one and obtain the average CMPE vector as $\overline{\mathrm{CMPE}}=\left[\overline{\mathrm{CMPE}}_{1}, \overline{\mathrm{CMPE}}_{2}, \ldots, \overline{\mathrm{CMPE}}_{s}\right]$, where $\overline{\mathrm{CMPE}}_{v}=k^{-1} \sum_{\nu=1}^{k} \mathrm{CMPE}_{\nu, v}$.

\section{Time-Frequency Characteristics}

Time-domain characteristic vectors are obtained by using a statistical analytical method to process the probability density function of OLTC vibration signals. Specifically, these signals can be divided into two types: dimensional and dimensionless. Two characteristic parameters of the response signal amplitude and energy are selected, which are the root mean square (RMS) error value $t_{1}$ and kurtosis $t_{2}$; six characteristic parameters of response signal time series distribution are selected: standard deviation $t_{3}$, peak-to-peak $t_{4}$, peak index $t_{5}$, pulse index $t_{6}$, margin index $t_{7}$, and waveform index $t_{8}$.

The frequency-domain characteristic parameter analysis refers to the transformation of the vibration signals from the time domain to the freqmethods. Compared with time-domain analysis, frequency-domain analysis provides more intuitive and rich fault information. Four characteristic parameters are selected to reflect the position change of the main frequency band: average frequency $p_{1}$, average frequentness $p_{2}$ (This transformation can facilitate further analysis and processing the signal by statistical fitness is a measure characterizing the dispersion of frequency components), waveform stability factor $p_{3}$, and center of gravity frequency $p_{4}$. Besides, three frequency-domain characteristic parameters are selected to reflect the dispersion and concentration degree of the spectrum, which are amplitude variance $p_{5}$, deviation degree $p_{6}$, and amplitude kurtosis $p_{7}$.

The time and frequency characteristics can be jointly expressed in the form of matrix as $\mathbf{b}_{1}=\left[t_{1}, t_{2}, \ldots, t_{8}\right]$ and $\mathbf{b}_{2}=$ $\left[p_{1}, p_{2}, \ldots, p_{7}\right]$. We can concatenate both matrices to yield the time-frequency characteristic matrix as $\mathbf{b}_{3}=\left[\mathbf{B}_{1}, \mathbf{B}_{2}\right]$. Consequently, all features are fused together to form a matrix incorporating all features, which is defined as follows:

$$
\mathbf{b}^{\prime}=\left[\mathbf{T}^{\prime}, \overline{\mathbf{C M P E}}, \mathbf{B}\right] .
$$

In this paper, the signal represented by $N$ samples is divided into $S$ segments. In particular, we stipulate that each segment has 1024 samples, and the feature matrix generated by $S$ segments can be expressed as

$$
\mathbf{B}^{\prime}=\left[\begin{array}{c}
\mathbf{b}_{1}^{\prime} \\
\mathbf{b}_{2}^{\prime} \\
\vdots \\
\mathbf{b}_{S}^{\prime}
\end{array}\right]=\left[\begin{array}{cccc}
b_{1,1}^{\prime} & b_{1,2}^{\prime} & \ldots & b_{1, J}^{\prime} \\
b_{2,1}^{\prime} & b_{2,2}^{\prime} & \ldots & b_{2, J}^{\prime} \\
\vdots & \vdots & \ddots & \vdots \\
b_{S, 1}^{\prime} & b_{S, 2}^{\prime} & \ldots & b_{S, J}^{\prime}
\end{array}\right]
$$

where $J=s+k+15$ is the dimension of the feasible solution and $S$ is thought of the number of whale populations in our specific application scenario.

\section{Feature Selection Model for OltC MECHANICAL FAULTS}

In this section, we first present the conventional whale optimization algorithm to clarify some basics and provide a comparison benchmark for our advanced algorithm. Then, we detail how the key features are selected to reduce the computational complexity and propose the IWOA-KNN algorithm that relies on the feature matrix to provide accurate fault diagnosis.

\section{A. Whale Optimization Algorithm}

The whale optimization algorithm is a new swarm intelligence optimization algorithm proposed by Mirjalili and Lewis [28]. By simulating the foraging behaviors of the bubble 
net, the whale optimization algorithm can be divided into three stages: encircling, spiral updating, and prey searching. First, whales search for prey and gradually obtain relevant information of prey. Then, whales constantly approach prey by surrounding and spiraling close to prey and finally pounce on prey. This process can also be transferred to find the optimal solution to an optimization problem. Accordingly, the three updating methods are formulated in the following paragraphs.

1) Surround food: As specified in [28], humpback whales swim to prey in a spiral motion when they are engaged in the predatory behavior of spitting bubbles. Therefore, the mathematical model of the spiral renewal stage can be expressed as follows

$$
\mathbf{M}_{1}(q+1)=\mathbf{M}^{*}(q)-A \mathbf{D}_{1},
$$

where $\mathbf{D}_{1}=\operatorname{Abs}\left(C \mathbf{M}^{*}(q)-\mathbf{M}(q)\right)$ is the distance matrix between the current optimal solution and the search body and $\operatorname{Abs}(\cdot)$ is the entry-wise absolute value conversion function; $q$ is the index of the current iteration; $\mathbf{M}^{*}(q)$ is the position matrix of the current optimal solution and $\mathbf{M}(q)$ is the position matrix of the search body; $A$ and $C$ are process control coefficients. Both process control coefficients are defined to be $A=a(2 r-1)$ and $C=2 r$, where the range of $a$ is $[0,2]$, and it is in the form of recursive subtraction updated with $a \leftarrow\left\{a-2 q / Q_{\max }\right\}$, in which $Q_{\max }$ represents the maximum number of iterations; $r$ is a random variable distributed over $[0,1]$.

2) Spiral renewal: Following the assumption given in [28], humpback whales swim to their prey in a spiral motion as a predator-prey behavior when they spit bubbles. Consequently, the mathematical model of the spiral renewal stage can be written as follows

$$
\mathbf{M}_{2}(q+1)=\mathbf{M}^{*}(q)+\mathbf{D}_{2} \exp (b l) \cos (2 \pi l),
$$

where $\mathbf{D}_{2}=\operatorname{Abs}\left(\mathbf{M}^{*}(q)-\mathbf{M}(q)\right)$ is the distance matrix between whale and prey; $b$ is a process control coefficient to regulate the shape of the spiral; $l$ is a random variable distributed within $(-1,1)$. Because whales not only swim towards a prey in a spiral motion, but also shrink the encirclement, the position update should be carried out depending on either (6) or (7) with a $50 \%$ probability as the threshold probability $\rho_{\mathrm{th}}$. The probabilistic expression is given by

$$
\mathbf{M}(q+1)=\left\{\begin{array}{ll}
\mathbf{M}_{1}(q+1), & \Delta<\rho_{\mathrm{th}} \\
\mathbf{M}_{2}(q+1), & \Delta \geq \rho_{\mathrm{th}}
\end{array},\right.
$$

where $\Delta$ is a random variable uniformly distributed over $[0,1]$.

3) Search prey: In addition to the bubble net search strategy, humpback whales can also search prey randomly, which is based on the value of process control coefficient $A$. If $A \notin$ $(-1,1)$, the distance matrix should be updated in a random manner. To find a prey, the whale will deviate from the original target prey, which makes the classic whale optimization algorithm have a global search ability. The random updating expression is given as follows

$$
\mathbf{M}(q+1)=\mathbf{M}_{\text {rand }}-A \mathbf{D}_{\text {rand }},
$$

where $\mathbf{D}_{\text {rand }}=\operatorname{Abs}\left(C \mathbf{M}_{\text {rand }}-\mathbf{M}(q)\right)$ and $\mathbf{M}_{\text {rand }}$ is the position of a random whale in the current population.

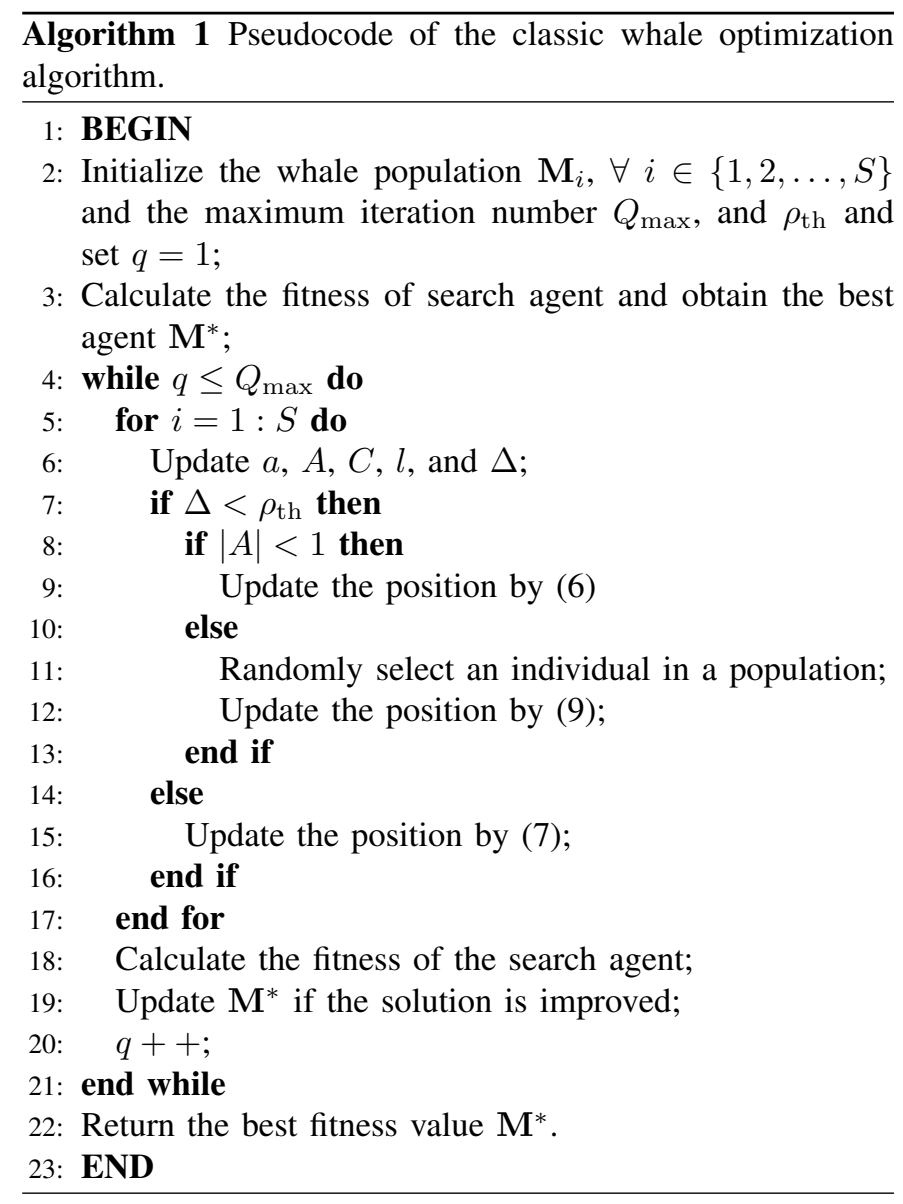

The pseudocode of the classic whale optimization algorithm is given in Algorithm 1 for clarification and comparison purposes.

\section{B. K-Nearest Neighbor Method}

The K-nearest neighbor (KNN) method is the simplest but one of the most effective non-parametric methods for classification and regression [29], especially when the size of the sample set is small. In the realm of machine learning, $\mathrm{KNN}$ is mainly used in regression analysis and modeling. In the process of classification modeling, the sample training, as well as the output of sample classification, rely on the KNN method. To enable the KNN based classification, the Euclidean distance between two arbitrary feature matrices $\mathbf{M}_{1}$ and $\mathbf{M}_{2}$ with the same dimension is defined as [30]

$$
\delta\left(\mathbf{M}_{1}, \mathbf{M}_{2}\right)=\left\|\mathbf{M}_{1}-\mathbf{M}_{2}\right\|_{\mathcal{F}},
$$

where $\|\cdot\|_{\mathcal{F}}$ is the Frobenius norm of the matrix enclosed.

When applying $\mathrm{KNN}$, it is common to divide datasets into training datasets and test datasets for training and verification purposes, respectively. Each sample in the test datasets needs to determine its $K$ nearest neighbors from the training data. To mitigate the overfitting problem, the cross-validation $(\mathrm{CV})$ method is introduced. In this paper, the $K$-fold $\mathrm{CV}$ method is used. In particular, the $K$-fold $\mathrm{CV}$ methods partitions the dataset into $K$ sets, among which $K-1$ sets are selected as a training group, and only a single set is used as a test 
dataset. The KNN classifier is trained by the $K-1$ datasets and then applied to the single remaining test dataset to predict the class labels of its samples. Subsequently, we can calculate the error prediction rate of the class label, which is also known as the classification error rate. The random results of different training rounds are averaged, and the reliable statistical results are hereby obtained. Without loss of generality, we set $K=10$ in this paper.

\section{Selection Model of OLTC Based on Multi-Feature Fusion and IWOA-KNN}

To enhance the accuracy of the mechanical fault feature identification for OLTCs, we propose a feature selection method based on multi-feature fusion and the joint application of an improved whale optimization algorithm (IWOA) and the KNN method (IWOA-KNN). Apparently, massive features will increase the complexity of feature recognition and reduce the accuracy of diagnosis. Therefore, it is necessary to perform feature selection before optimization. In this paper, We use the maximum correlation minimum redundancy (mRMR) principle to deal with the feature matrix $\mathbf{B}^{\prime}$ [26]. After the feature selection process, the reduced feature matrix is defined as follows:

$$
\mathbf{B}^{\prime \prime}=\left[\begin{array}{cccc}
b_{1,1}^{\prime \prime} & b_{1,2}^{\prime \prime} & \ldots & b_{1, F}^{\prime \prime} \\
b_{2,1}^{\prime \prime} & b_{2,2}^{\prime \prime} & \ldots & b_{2, F}^{\prime \prime} \\
\vdots & \vdots & \ddots & \vdots \\
b_{S, 1}^{\prime \prime} & b_{S, 2}^{\prime \prime} & \ldots & b_{S, F}^{\prime \prime}
\end{array}\right]
$$

where $F \leq J$ is the reduced dimension of the feasible solution.

With the reduced dimension of $F$, we can initialize the population position. The population position initialization based on the opposition based learning (OBL) algorithm can effectively improve the following optimization process [31], [32]. Therefore, we adopt this initialization approach in this paper. The population representation is given by

$$
\mathbf{H}^{+}=\left[\begin{array}{cccc}
h_{1,1}^{+} & h_{1,2}^{+} & \ldots & h_{1, F}^{+} \\
h_{2,1}^{+} & h_{2,2}^{+} & \ldots & h_{2, F}^{+} \\
\vdots & \vdots & \ddots & \vdots \\
h_{S, 1}^{+} & h_{S, 2}^{+} & \cdots & h_{S, F}^{+}
\end{array}\right]
$$

We can initialize each entry of $\mathbf{H}^{+}$by

$$
h_{i, j}^{+}=b_{i, j}^{\prime \prime}+\phi(i, j)\left(b_{\max , j}^{\prime \prime}-b_{\min , j}^{\prime \prime}\right),
$$

where $b_{\min , j}^{\prime \prime}=\min _{i \in\{1,2, \ldots, S\}}\left\{b_{i, j}^{+}\right\}$and $b_{\max , j}^{\prime \prime}=$ $\max _{i \in\{1,2, \ldots, S\}}\left\{b_{i, j}^{+}\right\} ; \phi(i, j)$ is an entry-wise random variable uniformly distributed over $[0,1]$. Similarly, we can determine the opposition population by

$$
\mathbf{H}^{-}=\left[\begin{array}{cccc}
h_{1,1}^{-} & h_{1,2}^{-} & \ldots & h_{1, F}^{-} \\
h_{2,1}^{-} & h_{2,2}^{-} & \ldots & h_{2, F}^{-} \\
\vdots & \vdots & \ddots & \vdots \\
h_{S, 1}^{-} & h_{S, 2}^{-} & \cdots & h_{S, F}^{-}
\end{array}\right]
$$

where

$$
h_{i, j}^{-}=b_{\min , j}+b_{\max , j}-h_{i, j}^{+} .
$$

Then, both population matrices can be concatenated to be

$$
\mathbf{H}=\left[\mathbf{H}^{+} ; \mathbf{H}^{-}\right]=\left[\begin{array}{cccc}
h_{1,1}^{+} & h_{1,2}^{+} & \ldots & h_{1, F}^{+} \\
h_{2,1}^{+} & h_{2,2}^{+} & \ldots & h_{2, F}^{+} \\
\vdots & \vdots & \ddots & \vdots \\
h_{S, 1}^{+} & h_{S, 2}^{+} & \ldots & h_{S, F}^{+} \\
h_{1,1}^{-} & h_{1,2}^{-} & \ldots & h_{1, F}^{-} \\
h_{2,1}^{-} & h_{2,2}^{-} & \ldots & h_{2, F}^{-} \\
\vdots & \vdots & \ddots & \vdots \\
h_{S, 1}^{-} & h_{S, 2}^{-} & \ldots & h_{S, F}^{-}
\end{array}\right] .
$$

Subsequently, we need to keep the dimension of the population and halve the concatenated matrix to obtain. To do so, we first introduce the fitness function of the classification process. Because the feature selection is considered as a multiobjective problem, it is necessary to simultaneously minimize the number of features and maximize the classification accuracy of a given classifier. As a result, the fitness function can be defined infra

$$
\text { Fitness }=\alpha \gamma+(1-\alpha) \xi / F,
$$

where $\alpha \in[0,1]$ is a weighting parameter, which is used to balance between the classifiers accuracy and the number of the selected features; $\gamma$ is error rate which is calculated by using the KNN classifier; $\xi$ is the number of feature subsets. From the above definition of the fitness function, it is clear that the value range of Fitness is $[0,1]$. By the fitness function defined in (17), we can construct the fitness vector as $\mathbf{F}=$ $\left[\right.$ Fitness $_{1}$, Fitness $_{2}, \ldots$, Fitness $\left._{2 S}\right]$, corresponding to the rows of $\mathbf{H}$. We sort the elements of $\mathbf{F}$ in ascending order and obtain the sorted vector $\mathbf{F}_{\text {sort }}$. We can obtain the original indexes of the first $S$ elements of $\mathbf{F}_{\text {sort }}$ and extract the row vectors with the corresponding indexes in $\mathbf{H}$ to form a new matrix $\mathbf{G}$. Finally, we quantize the element in $\mathbf{G}$ by the following rule:

$$
m=\mathbb{I}\{g>0.5\},
$$

where $\mathbb{I}\{\cdot\}$ is the the Iverson bracket that returns 1 if the enclosed condition is true or 0 otherwise [33]. This operation converts continuous values to binary values, i.e., 0 or 1 .

By the entry-wise quantization over $\mathbf{G}$, we can obtain the initial population matrix

$$
\mathbf{M}(0)=\left[\begin{array}{cccc}
m_{1,1}(0) & m_{1,2}(0) & \ldots & m_{1, F}(0) \\
m_{2,1}(0) & m_{2,2}(0) & \ldots & m_{2, F}(0) \\
\vdots & \vdots & \ddots & \vdots \\
m_{S, 1}(0) & m_{S, 2}(0) & \ldots & m_{S, F}(0)
\end{array}\right] .
$$

We present the pseudocode of the entire initialization process in Algorithm 2.

To obtain an adequate equilibrium between search space and optimization efficiency, we introduce a dynamic inertia weight to improve the position update relation, which is given as follows [31]:

$$
\omega=\omega_{\max }-\left(\omega_{\max }-\omega_{\min }\right)\left(q / Q_{\max }\right)^{1 / q},
$$

where $\omega_{\max }$ and $\omega_{\min }$ are the maximum and minimum inertia weights, respectively. When $\lambda<\rho_{\text {th }}$ and $|A|<1$, we then 


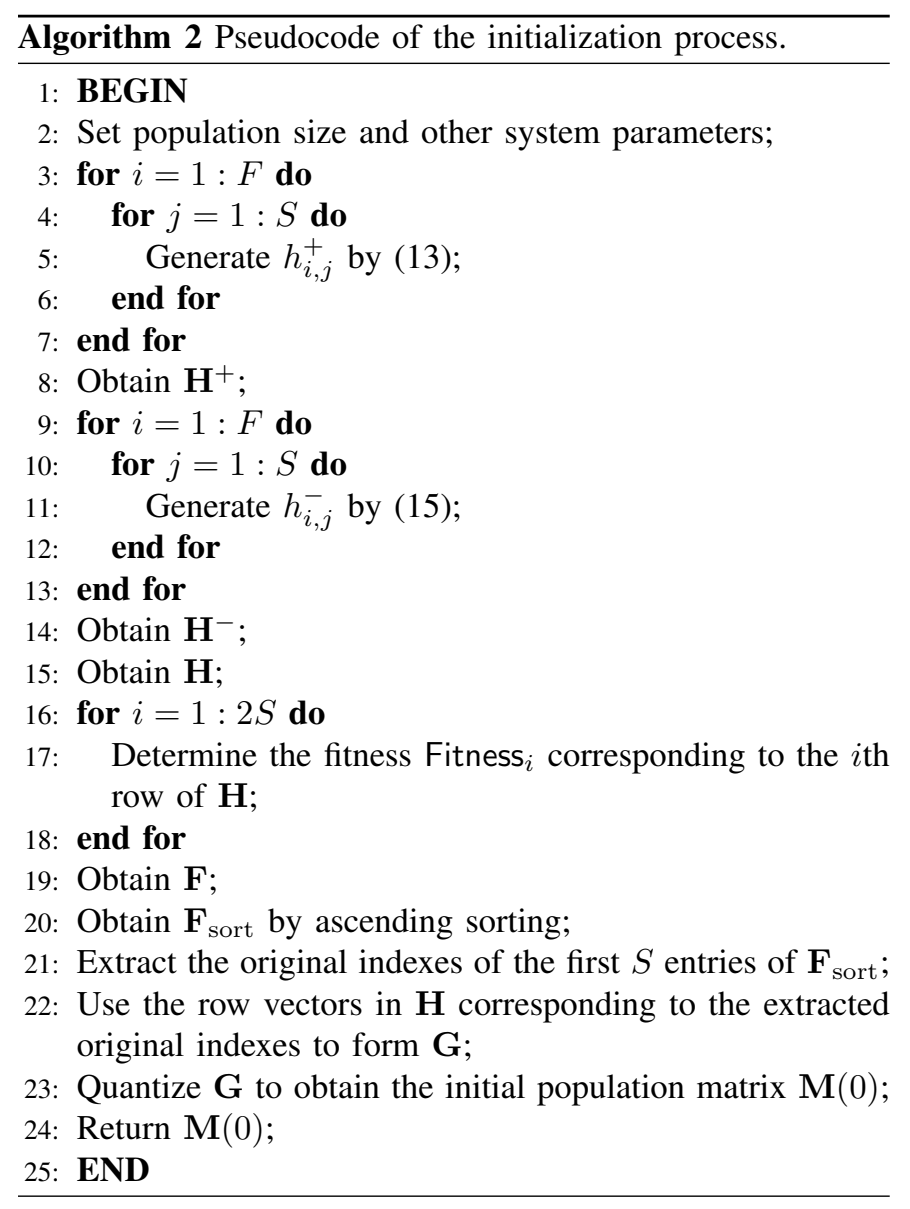

update the whale positions by

$$
\begin{aligned}
\mathbf{M}^{\omega}(q+1)= & M_{1}^{\omega}(q+1) \mathbb{I}\left\{\lambda<\rho_{\mathrm{th}}\right\} \mathbb{I}\{|A|<1\} \\
& +M_{\text {rand }}^{\omega}(q+1) \mathbb{I}\left\{\lambda<\rho_{\mathrm{th}}\right\} \mathbb{I}\{|A| \geq 1\} \\
& +M_{2}(q+1) \mathbb{I}\left\{\lambda \geq \rho_{\mathrm{th}}\right\},
\end{aligned}
$$

where

$$
M_{1}^{\omega}(q+1)=\mathbf{M}^{*}(q)-\omega A \mathbf{D}_{1},
$$

and

$$
M_{\text {rand }}^{\omega}(q+1)=\mathbf{M}_{\text {rand }}-\omega A \mathbf{D}_{\text {rand }} .
$$

The pseudocode of the IWOA is presented in Algorithm 3 for clarification and comparison purposes. The overall IWOAKNN optimization process is pictorially illustrated in Fig. 1.

\section{EXPERIMENTAL Verification AND Discussion}

In this section, we present the experimental results to verify the proposed IWOA-KNN method for fault diagnosis and discuss the results in detail. We also give the details of the experiment setup and the approaches to extracting various features.

\section{A. Experiment Setup}

In this paper, the model CM111-50-63B-10193W Huaming M-type on-load tap changer is used. The type of sensor is LC0151 based on the integration of traditional piezoelectric sensors and charge amplifiers. The sampling frequency is 50

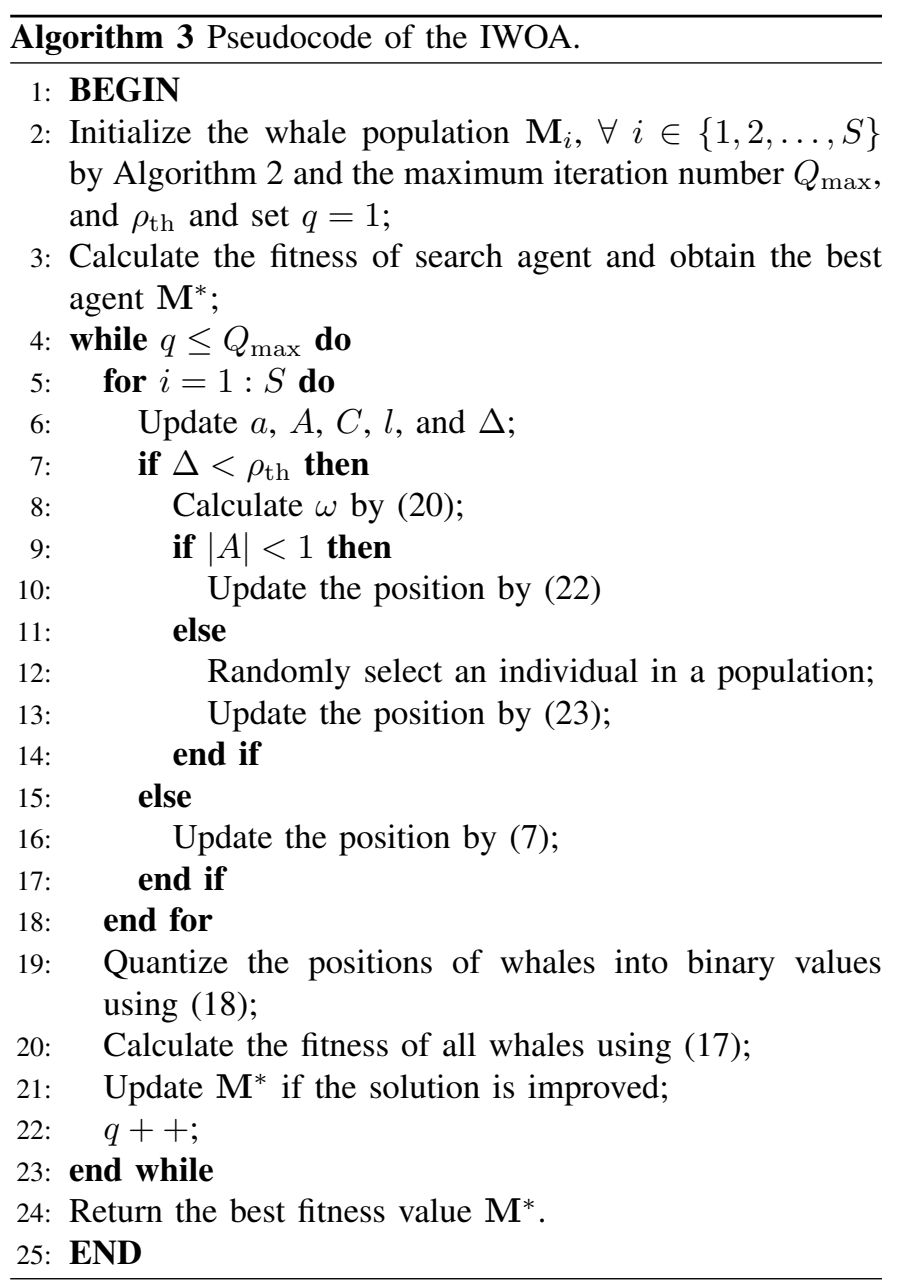

$\mathrm{kHz}$. Under normal working conditions the shifting process of M-type OLTC is about 140-150 ms. The frequency is concentrated within $20 \mathrm{kHz}$, and 7830 sampling points are thereby selected. The acceleration sensor is installed near the vibration source, which is comprehensively considered to be on the top of the on-load tap changer, and the vibration signal is converted into a digital signal through the data acquisition card and stored on a laptop computer. The installation diagram and test system diagram of the sensor is shown in Fig. 2. Through the tripod hoisting, the action mechanism is extracted from the inside of the tap changer to set the fault of the contact. In the process of contact replacement, due to the heavy weight of the switch core, it is necessary to lift the switch core from the oil chamber of the tap changer by a tripod crane ${ }^{1}$.

In this paper, four kinds of vibration signals yielded by the OLTCs can be identified and analyzed in the experiments, which are corresponding to normal operation, contact burning, contact loosening, and contact shedding. To analyze the measured signal waveform, first, the signal is normalized, and the average amplitude under normal working conditions is taken as the benchmark. Similarly, the signal amplitudes under other working conditions multiple the same normalization coefficient. In this way, the average vibration amplitude under different working conditions is consistent. It should be noted

\footnotetext{
${ }^{1}$ The details of the lifting procedure are given in [34]
} 


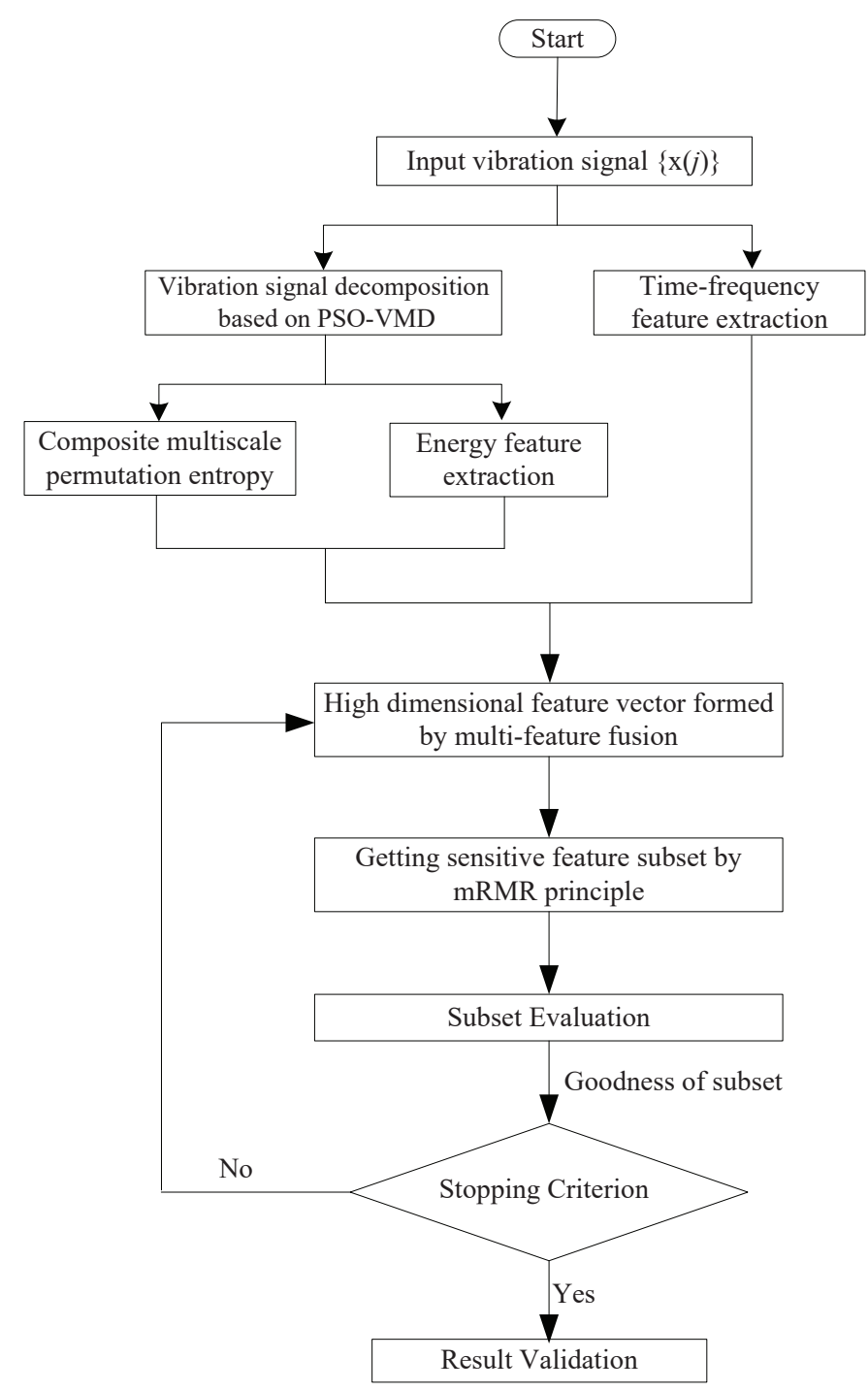

Fig. 1: Flowchart of the overall IWOA-KNN optimization process.

that the normalization process only changes the amplitude of the signal, rather than the influence of the amplitude on the results. Therefore, the integrity and the contained fault information of the detected signals can be retained. We illustrated the amplitudes of the four normalized signals in Fig. 3.

\section{B. Extraction of Multi-Feature Parameters}

In this paper, taking the vibration signal of normal conditions as an example. The value of $E_{p}$ is obtained by decomposing the vibration signal of normal working conditions using the PSO-VMD method. We present the minimum of $E_{p}$ in terms of the number of iterations in Fig. 4. As can be seen from Fig. 4, after the 7 th iteration, minimum $E_{p}$ begins to converge, so the double parameter value of VMD optimized by the PSO algorithm is $[K, \Psi]=[7,893]$. The time-domain diagram and frequency-domain spectrum diagram of IMFs obtained by the PSO-VMD decomposition is illustrated in Fig. 5. From this figure, it is obvious that the VMD can decompose the vibration signal from low frequency to high frequency.

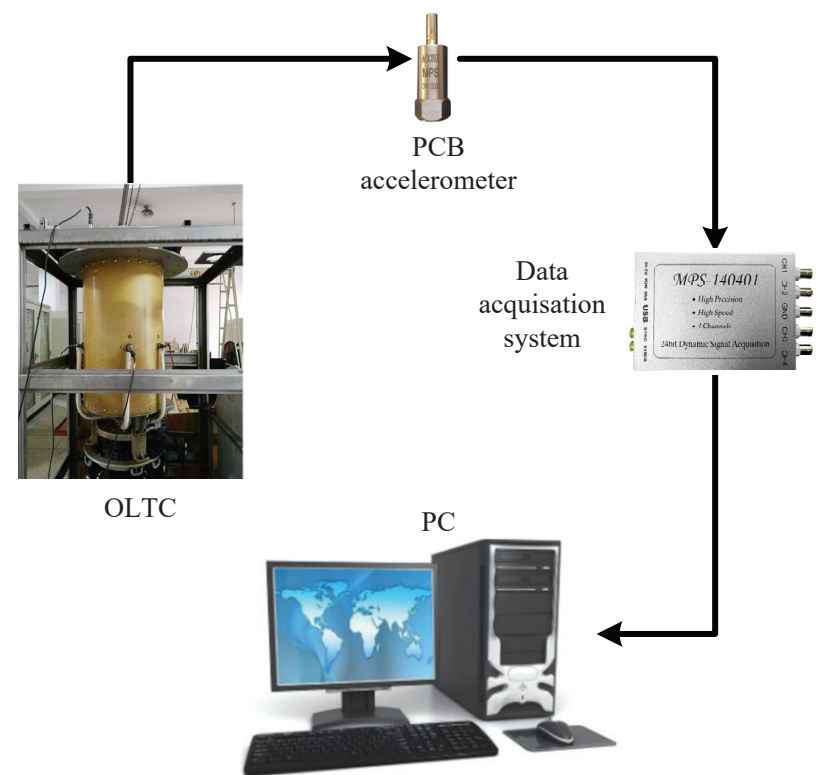

Fig. 2: Schematic diagram of OLTC vibration test system.

The distribution of specific energy characteristics of four OLTC vibration signals is shown in Fig. 6. The abscissa represents different IMFs, and the ordinate represents energy. By the results given in this figure, we verify that energy characteristics can classify different types of vibration signals from OLTCs. The results also show that the energy distribution can be used as a reference for fault feature selection for OLTCs.

The CMPE value corresponding to each IMF after decomposition is taken as a multi-scale feature of the vibration signal. The CMPE value is related to the number of samples $N$, scale factor $s$, embedding dimension $\eta$, and delay time $\tau$. Considering that the composite multi-scale permutation entropy is an improvement of the multi-scale permutation entropy, we set the system parameters as $N=7280, \eta=$ $3,4,5,6$, and $s=20$. We average the value for ten times and present them in Fig. 7. In figure 7, the abscissa represents the scale factor $\eta$ and the ordinate represents the CMPE value. It can be seen from this figure, for all cases with different $\eta$, four different operations conditions can be distinguished well.

In this paper, the time-frequency features of four normalized OLTC vibration signals are determined by the methods proposed in [35], [36] and constructed in the matrix form as specified in Section II. In this way, $\mathbf{B}^{\prime}$ for our experiments can be obtained.

\section{Experimental Results and Discussion}

In order to verify the method in this paper, we adopt a set of evaluation metrics for experiments, including the mean, standard deviation (STD), maximum, and minimum of fitness. The mean and STD of fitness can be expressed as

$$
\text { Mean }=\frac{1}{R} \sum_{r=1}^{R} \text { Fitness }_{r},
$$



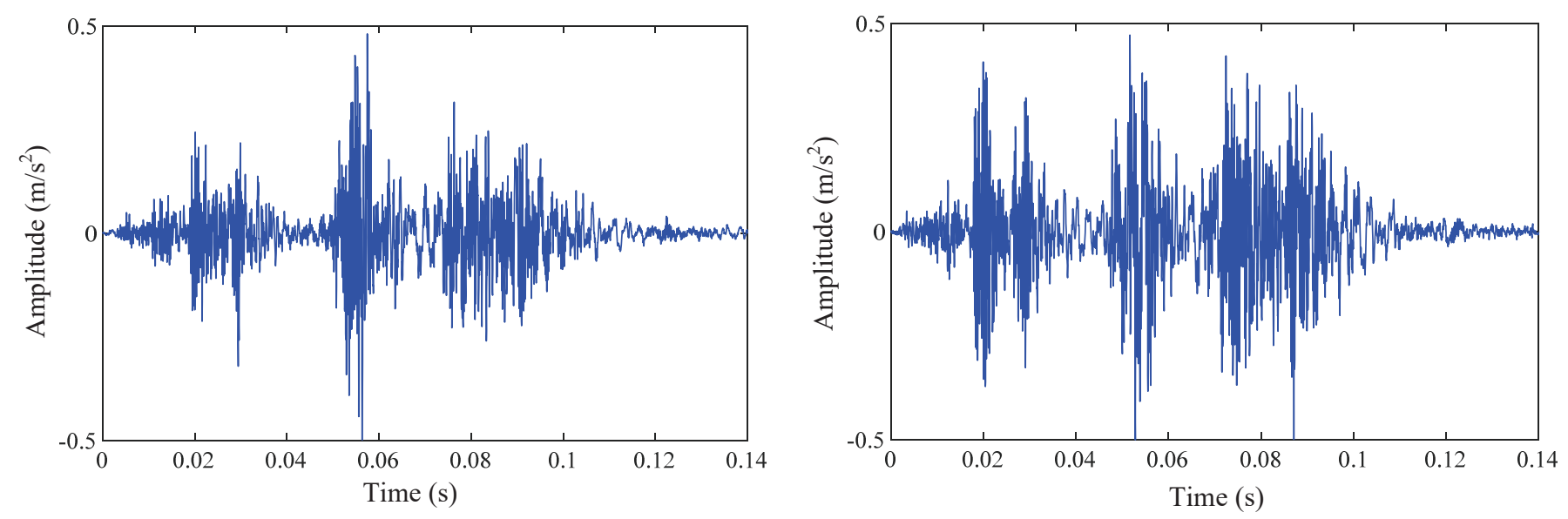

(a) Normal condition.
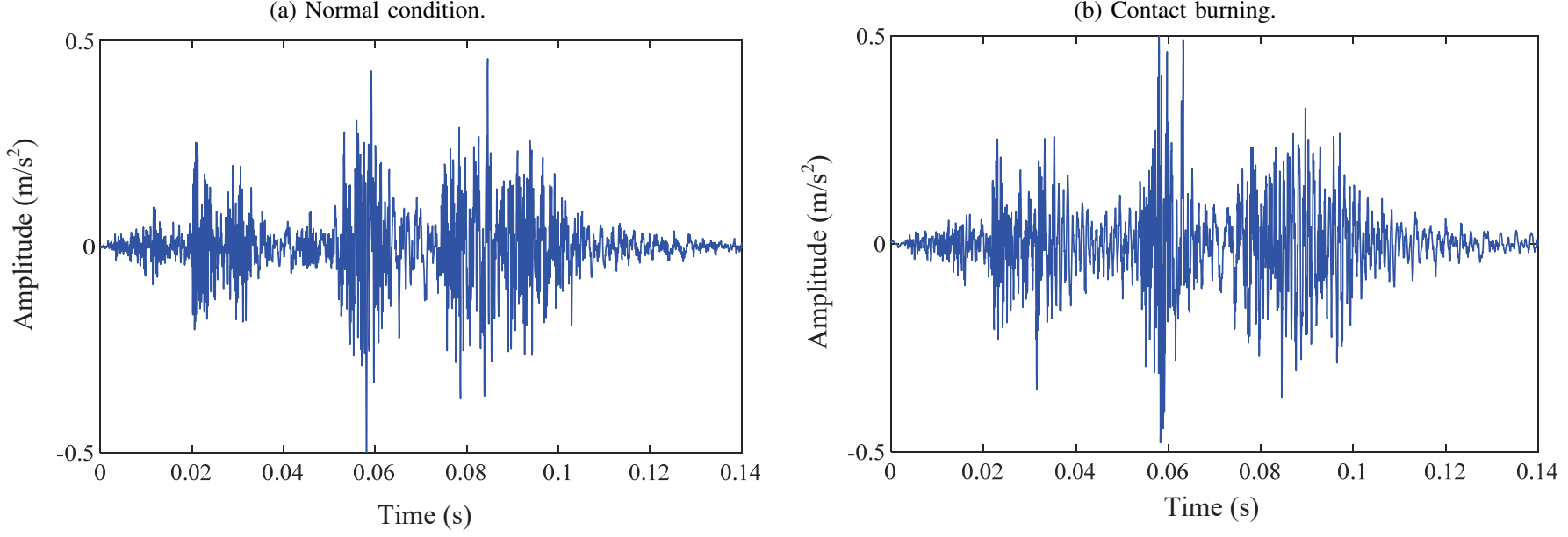

(c) Contact loosening.

(d) Contact shedding.

Fig. 3: Vibration signals corresponding to four different operational states of OLTCs.

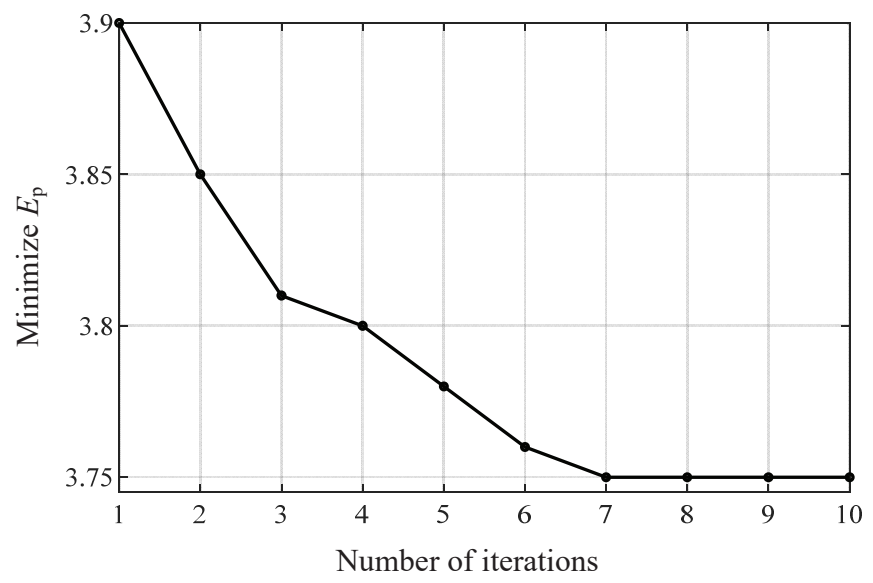

Fig. 4: Minimum $E_{p}$ versus the number of iterations.

and

$$
\mathrm{STD}=\left(\frac{1}{R} \sum_{r=1}^{R}\left(\text { Fitness }_{r}-\text { Mean }\right)\right)^{1 / 2} .
$$

To verify the effectiveness of the proposed multi-feature fusion-based method, we compare its performance with several crucial benchmarks, including the single composite multi-scale permutation entropy (SCMPE), feature fusion based multiscale fuzzy entropy (FFMFE), and single multi-scale fuzzy entropy (SMFE) [37], [38]. For each working condition, 50 data groups are collected and processed. Each data group consists of 7280 samples. 10-fold cross-validation (CV) methods for splitting partitions the dataset into 10 sets, of which 9 sets are randomly selected for training purposes and the one remaining set is used for testing purposes. To facilitate the experiments testing CMPE, we introduce another two parameters, which are the number of $\mathrm{KNN}$ classifications $c=5$ and the maximum scale factor $s_{\max }=20$. We follow the suggestion from [39] and let the algorithmic weighting parameter for fitness $\alpha=0.01$ so as to achieve an appropriate feature selection recognition rate. In addition, we set other algorithmic parameters as $\omega_{\max }=0.9, \omega_{\min }=0.4, \tau=1$, $Q_{\max }=100$, and $S=42$.

Fig. 8 and Fig. 9 show the means and STDs for different fault diagnosis methods obtained from 50 trials. In these experiments, we employed the mRMR principle to screen the sensitive feature subsets. It can be seen from Fig. 8 that the mean of fitness regarding the proposed method is 


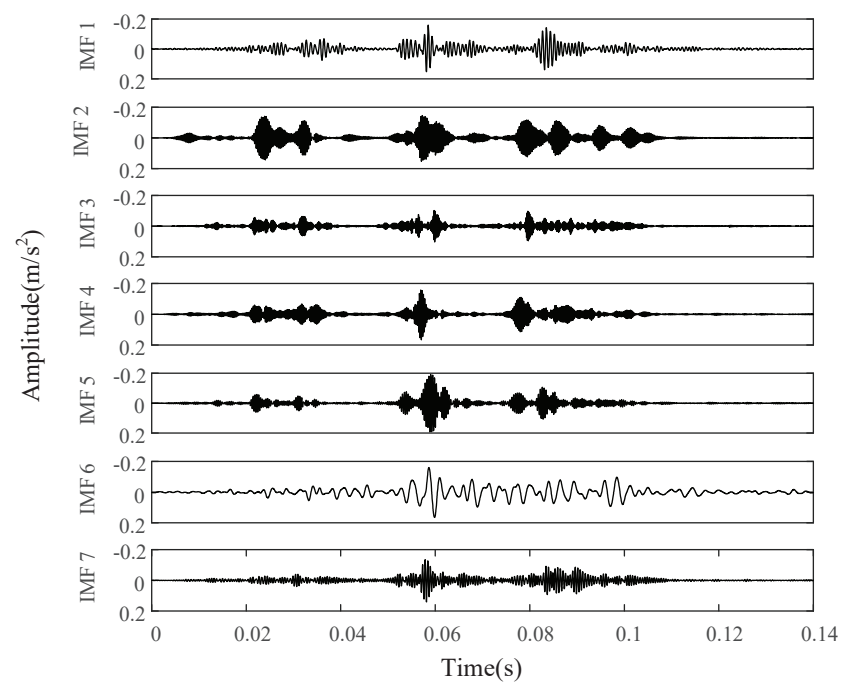

(a) Time-domain diagram of IMFs.

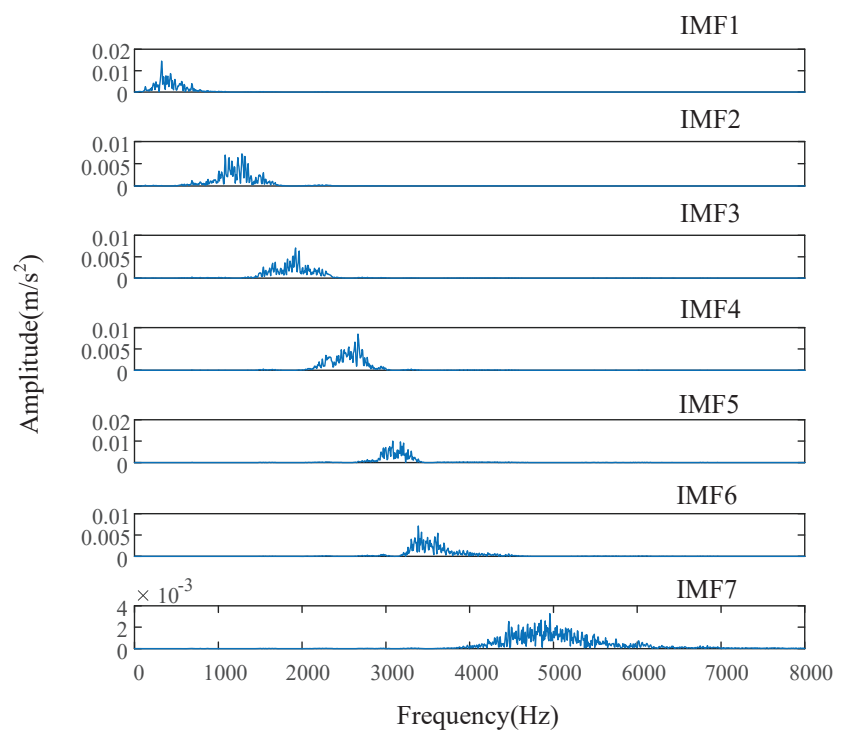

(b) Frequency-domain spectrum diagram of IMFs.

Fig. 5: Decomposition result of PSO-VMD.

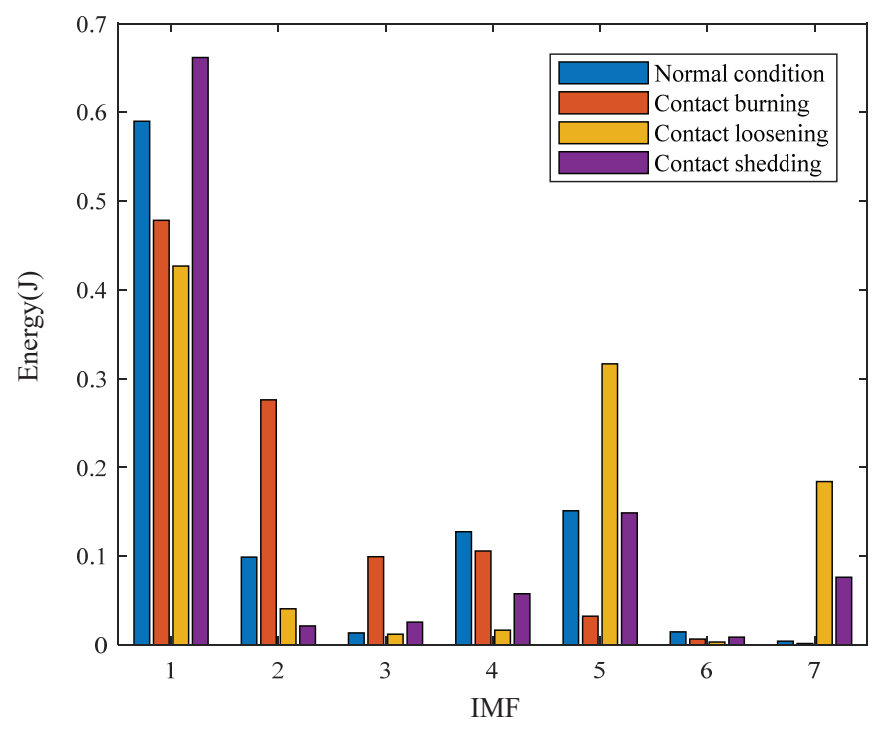

Fig. 6: Energy characteristics of IMFs.

the highest among all cases. This implies that the proposed method has a better diagnostic capability than all benchmarks. The comparison of the mean of fitness verifies the diagnostic advantages of the proposed method for mechanical faults of OLTCs.Moreover, as shown in Fig. 9, the STD of the proposed method is the lowest among all methods, which indicates a good optimization reliability.

To study the necessity of the mRMR principle for our proposed diagnostic method, we carry out experiments using the same system parameters for the six diagnostic methods without introducing the mRMR principle. The experimental results of mean and STD are shown in Fig. 10 and Fig. 11, respectively. From both figures, we can clearly see that the performance advantage reflected by the mean of fitness has been kept without introducing the mRMR principle. However, the reliability advantage might not necessarily be maintained always, since the STD corresponding to the proposed method get higher than the benchmarks for some cases. The comparison between the experiments with and without utilizing the mRMR principle verify that this principle is imperative for keeping the optimized results reliable.

In addition, given $\eta=4$, we also conduct experiments with the same settings as above to compare IWOA-KNN to the benchmarks with comparable settings: whale optimization algorithm (WOA)-KNN, discrete PSO (DPSO)-KNN [40], PSOKNN [41], and grasshopper optimization algorithm (GOA)KNN [42]. The experimental results of mean and deviation are shown in Fig. 12 and Fig. 13. The results of mean substantiate that IWOA-KNN outperforms the four benchmarks, and the results of STD show the reliability advantage of IWOA-KNN over the four benchmarks.

\section{CONCLUSION}

In this paper, multi-feature fusion and IWOA were employed to select mechanical fault features of OLTCs. Also, the mRMR principle was used to filter the high-dimensional feature set encompassing time-domain and frequency-domain characteristics as well as energy and composite multi-scale permutation entropy. In addition, IWOA was used to optimize the sensitive feature subset to obtain the optimal feature subset, and KNN was used to classify the different types of optimal feature subset. Compared with a number of benchmarks for four different OLTC working conditions, the proposed method yielded the best performance. The experimental data analysis and comparison verified the effectiveness of the proposed method. Moreover, the advantages of CMPE based feature fusion in extracting sensitive features were corroborated, which showed the necessity of the mRMR principle. The high-accuracy nature of the proposed method is expected to accelerate the promotion of EVs and the establishment of intelligent transportation environments. 

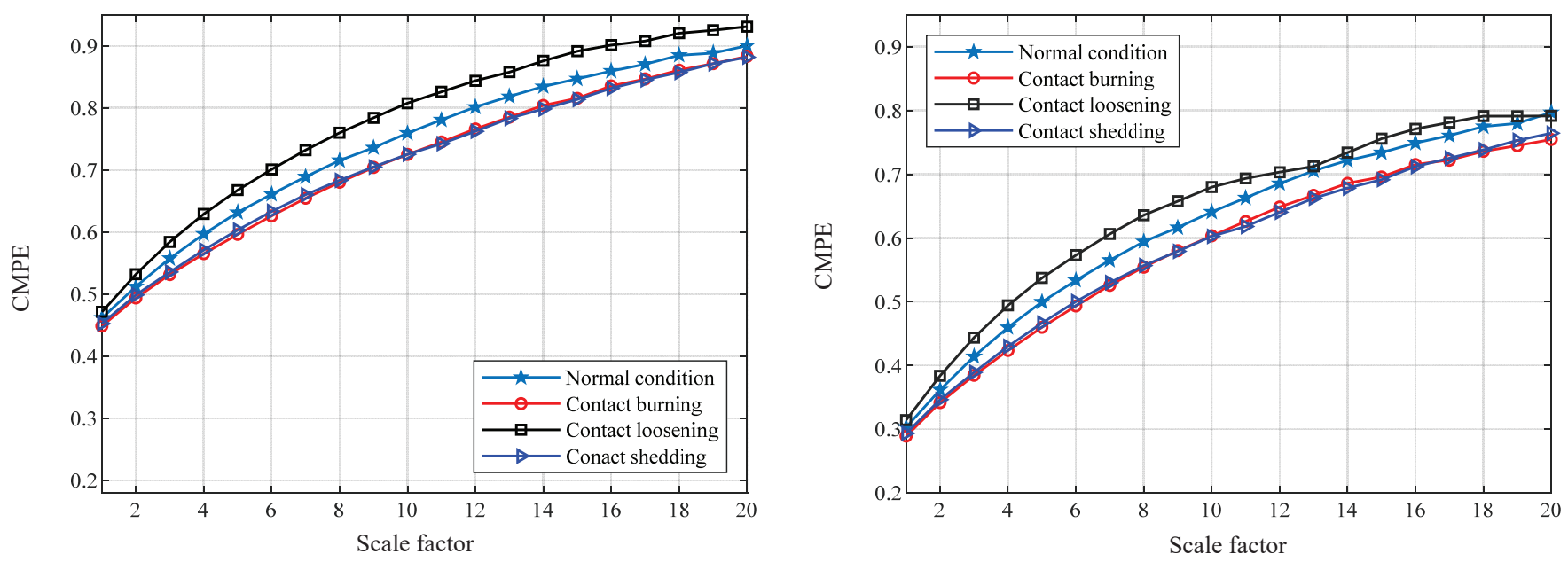

(a) $\eta=3$.

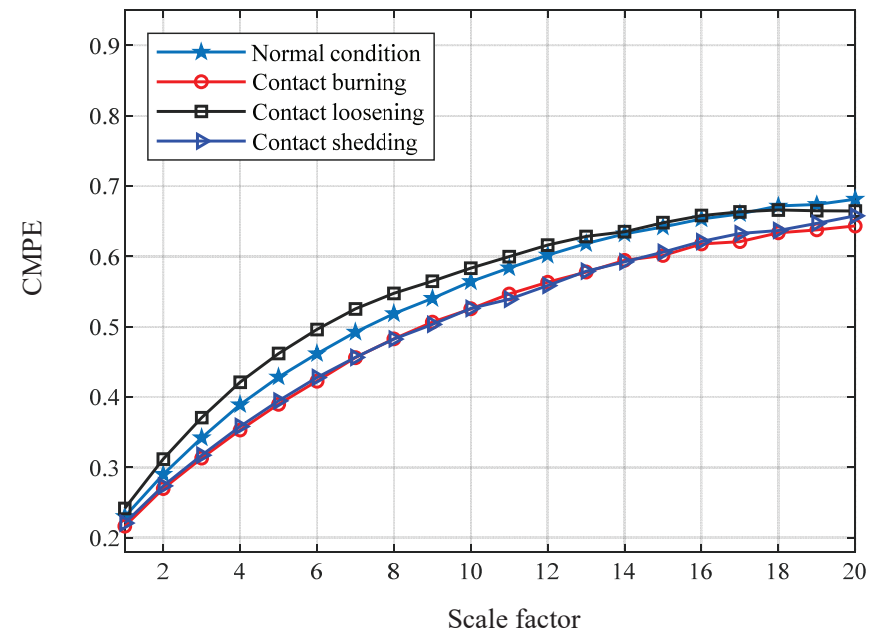

(b) $\eta=4$.

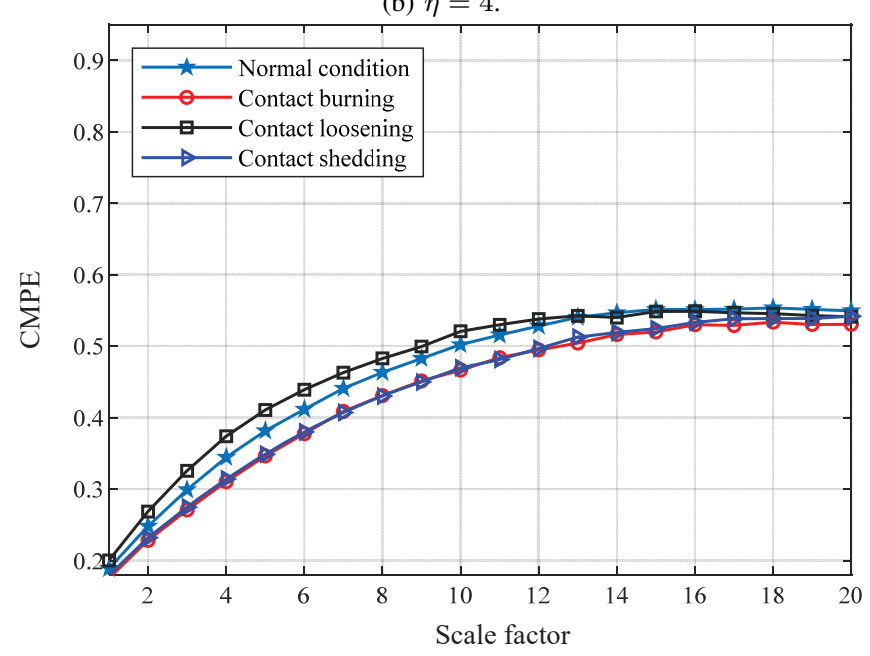

(c) $\eta=5$.

(d) $\eta=6$.

Fig. 7: Relation between $\eta$ and CMPE for different operational states of OLTCs.

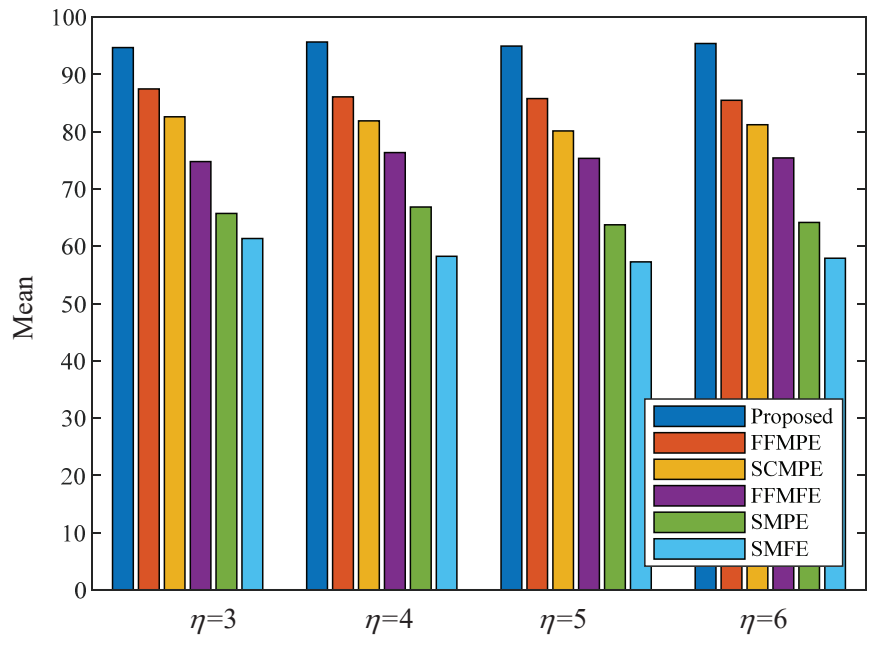

Fig. 8: Means corresponding to different values of $\eta$ for different algorithms with mRMR.

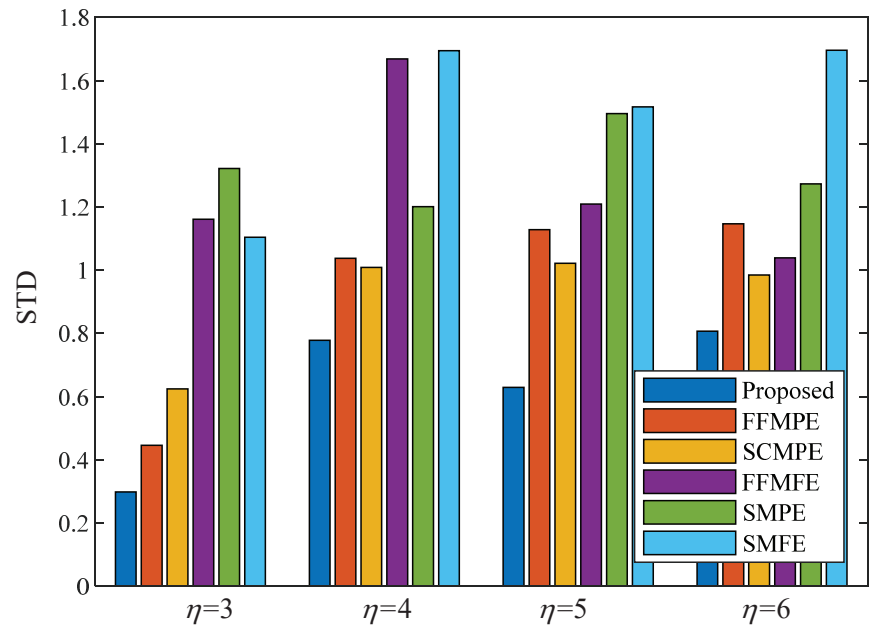

Fig. 9: STDs corresponding to different values of $\eta$ for different algorithms with mRMR. 


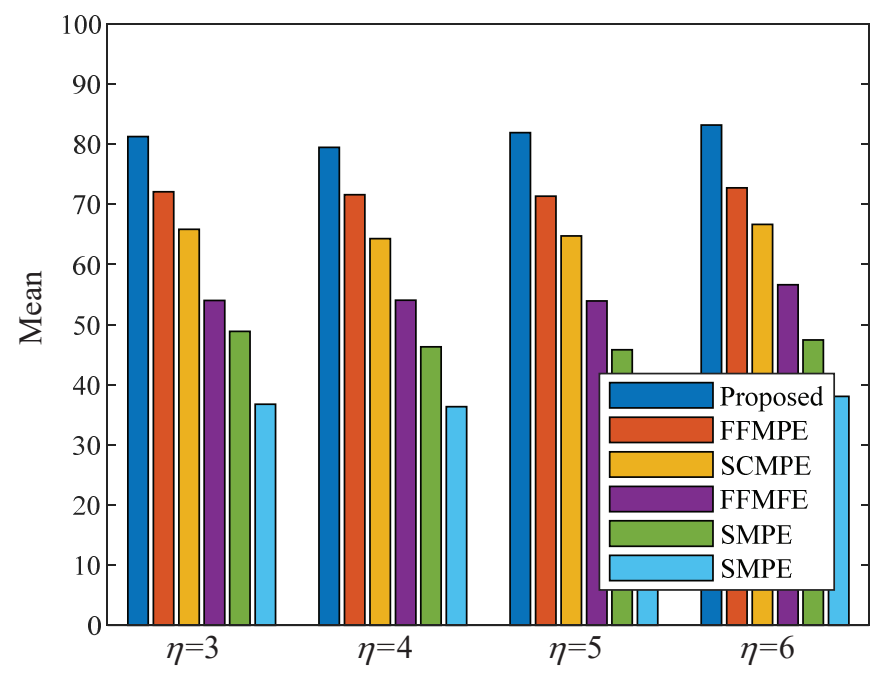

Fig. 10: Means corresponding to different values of $\eta$ for different algorithms without mRMR.

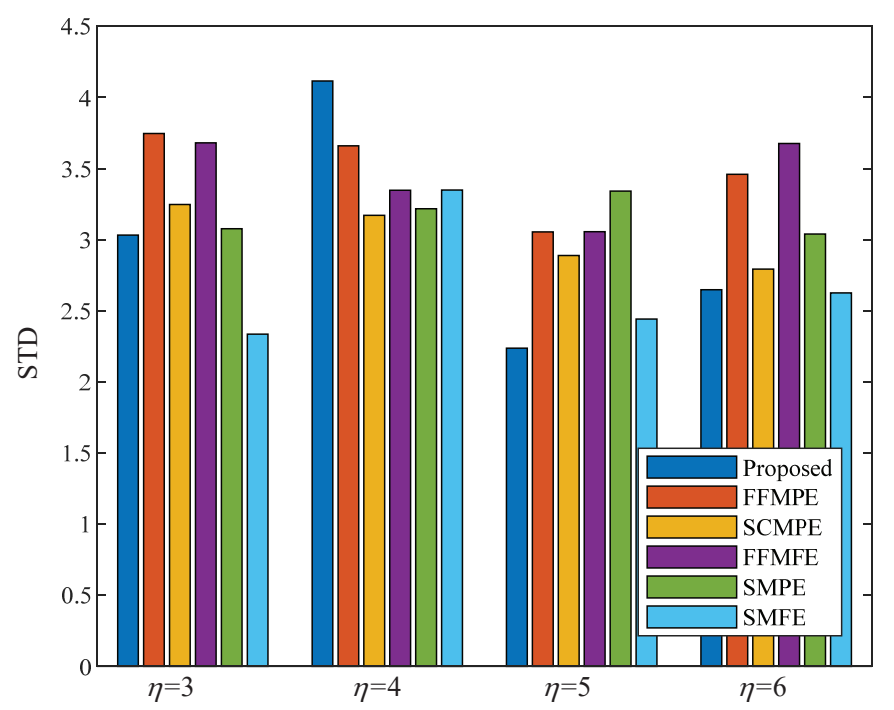

Fig. 11: STDs corresponding to different values of $\eta$ for different algorithms without mRMR.

\section{RefERENCES}

[1] R. Duan and F. Wang, "Fault diagnosis of on-load tap-changer in converter transformer based on time-frequency vibration analysis," IEEE Transactions on Industrial Electronics, vol. 63, no. 6, pp. 3815-3823, 2016.

[2] Y. Xu, C. Zhou, J. Geng, S. Gao, and P. Wang, "A method for diagnosing mechanical faults of on-load tap changer based on ensemble empirical mode decomposition, volterra model and decision acyclic graph support vector machine," IEEE Access, vol. 7, pp. 84 803-84 816, 2019.

[3] S. Dang, A. Odonde, T. Mirza, C. Dissanayake, and R. Burns, "Sustainable energy management: An analysis report of the impacts of electric vehicles," in Proc. IEEE EEEIC, Krakow, Poland, 2014, pp. 318-322.

[4] I. A. Metwally, "Failures, monitoring and new trends of power transformers," IEEE Potentials, vol. 30, no. 3, pp. 36-43, 2011.

[5] E. F. Simas F., L. A. L. de Almeida, and A. C. de C. Lima, "Vibration monitoring of on-load tap changers using a genetic algorithm," in Proc. IEEE Instrumentationand Measurement Technology Conference Proceedings, vol. 3, Ottawa, Canada, 2005, pp. 2288-2293.

[6] R. Yang, D. Zhang, Z. Li, K. Yang, S. Mo, and L. Li, "Mechanical fault diagnostics of power transformer on-load tap changers using dynamic

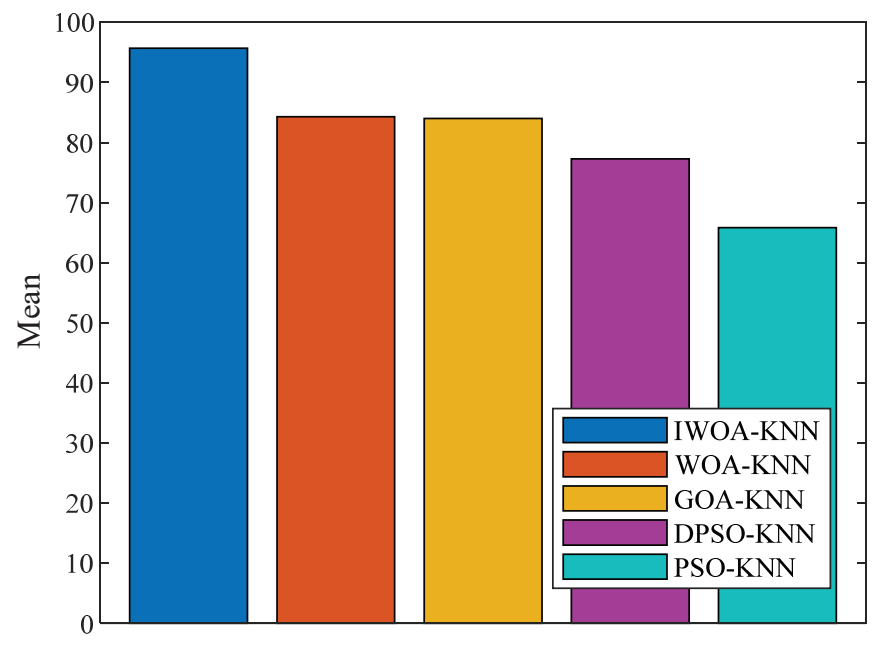

Fig. 12: Means corresponding to different optimization schemes, given $\eta=4$.

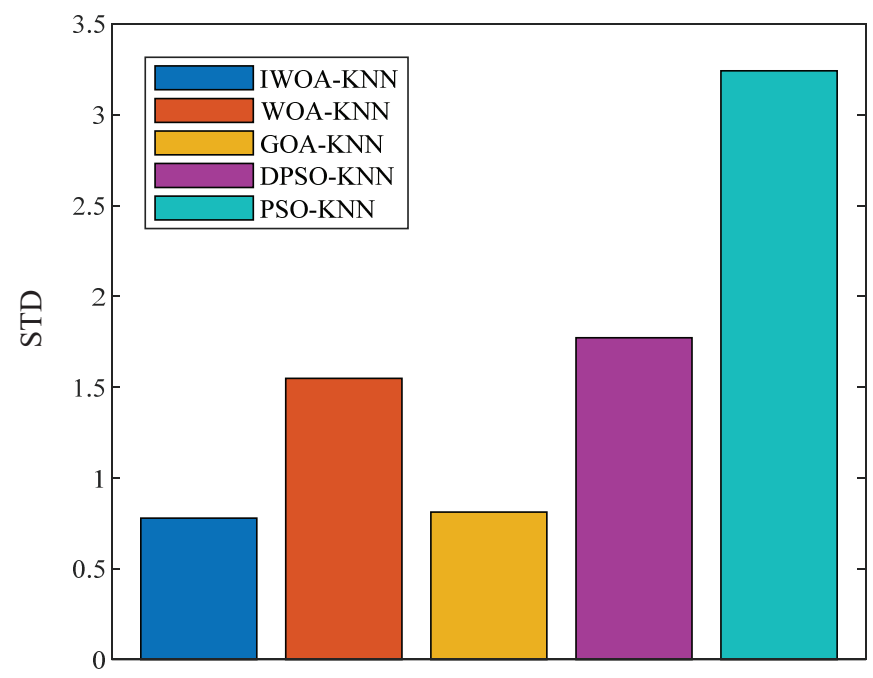

Fig. 13: STDs corresponding to different optimization schemes, given $\eta=4$.

time warping," IEEE Transactions on Instrumentation and Measurement, vol. 68, no. 9, pp. 3119-3127, 2019.

[7] C. Bengtsson, "Status and trends in transformer monitoring," IEEE Transactions on Power Delivery, vol. 11, no. 3, pp. 1379-1384, 1996.

[8] P. Kang and D. Birtwhistle, "Condition monitoring of power transformer on-load tap-changers. Part 1: Automatic condition diagnostics," IEE Proceedings-Generation, Transmission and Distribution, vol. 148, no. 4, pp. 301-306, 2001.

[9] _ - "Condition monitoring of power transformer on-load tap-changers. Part 2: Detection of ageing from vibration signatures," IEE ProceedingsGeneration, Transmission and Distribution, vol. 148, no. 4, pp. 307-311, 2001.

[10] Pengju Kang and D. Birtwhistle, "Condition assessment of power transformer onload tap changers using wavelet analysis and self-organizing map: Field evaluation," IEEE Transactions on Power Delivery, vol. 18, no. 1, pp. 78-84, 2003.

[11] J. Seo, H. Ma, and T. K. Saha, "A joint vibration and arcing measurement system for online condition monitoring of onload tap changer of the power transformer," IEEE Transactions on Power Delivery, vol. 32, no. 2, pp. 1031-1038, 2017. 
[12] T. Zhao, L. Zhang, and Q.-M. Li, "Feature analysis methodology for phase portrait structure of mechanical vibration signals of on-load tap changers in multidimensional space," Gaodianya Jishu/ High Voltage Engineering, vol. 33, no. 8, pp. 102-105, 2007.

[13] Q. Li, T. Zhao, L. Zhang, and J. Lou, "Mechanical fault diagnostics of onload tap changer within power transformers based on hidden Markov model," IEEE Transactions on Power Delivery, vol. 27, no. 2, pp. 596601, 2012.

[14] M. Abbasi, H. Rezaei, V. G. Menon, L. Qi, and M. R. Khosravi, "Enhancing the performance of flow classification in SDN-based intelligent vehicular networks," IEEE Transactions on Intelligent Transportation Systems, pp. 1-10, 2020.

[15] S. Dang, O. Amin, B. Shihada, and M.-S. Alouini, "What should 6G be?" Nature Electronics, vol. 3, no. 1, pp. 20-29, 2020.

[16] Y. Yang, D. Fei, and S. Dang, "Inter-vehicle cooperation channel estimation for IEEE 802.11p V2I communications," Journal of Communications and Networks, vol. 19, no. 3, pp. 227-238, 2017.

[17] Y. Yang, S. Dang, Y. He, and M. Guizani, "Markov decision-based pilot optimization for $5 \mathrm{G} \mathrm{V} 2 \mathrm{X}$ vehicular communications," IEEE Internet of Things Journal, vol. 6, no. 1, pp. 1090-1103, 2019.

[18] M. Abbasi, M. Rafiee, M. R. Khosravi, A. Jolfaei, V. G. Menon, and J. M. Koushyar, "An efficient parallel genetic algorithm solution for vehicle routing problem in cloud implementation of the intelligent transportation systems," Journal of Cloud Computing, vol. 9, no. 1, pp. 1-14, 2020.

[19] S. Jacob, V. G. Menon, S. P G, F. S. KS, B. Mahapatra, and S. Joseph, "Bidirectional multi-tier cognitive swarm drone 5G network," pp. 12191224, 2020.

[20] S. Dang, M. Wen, S. Mumtaz, J. Li, and C. Li, "Enabling multi-carrier relay selection by sensing fusion and cascaded ANN for intelligent vehicular communications," IEEE Sensors Journal, 2020.

[21] J. Li, S. Dang, Y. Yan, Y. Peng, S. Al-Rubaye, and A. Tsourdos, "Generalized quadrature spatial modulation and its application to vehicular networks with NOMA," IEEE Transactions on Intelligent Transportation Systems, 2020.

[22] W. Yang, Z. Peng, K. Wei, P. Shi, and W. Tian, "Superiorities of variational mode decomposition over empirical mode decomposition particularly in time-frequency feature extraction and wind turbine condition monitoring," IET Renewable Power Generation, vol. 11, no. 4, pp. 443452, 2016.

[23] K. Dragomiretskiy and D. Zosso, "Variational mode decomposition," IEEE Transactions on Signal Processing, vol. 62, no. 3, pp. 531-544, 2014.

[24] X. Zhang, Q. Miao, H. Zhang, and L. Wang, "A parameter-adaptive VMD method based on grasshopper optimization algorithm to analyze vibration signals from rotating machinery," Mechanical Systems and Signal Processing, vol. 108, pp. 58-72, 2018.

[25] J. Kennedy and R. Eberhart, "Particle swarm optimization," in Proc. IEEE International Conference on Neural Networks, vol. 4, Perth, WA, Australia, 1995, pp. 1942-1948 vol.4.

[26] G. Tang and X. Wang, "Parameter optimized variational mode decomposition method with application to incipient fault diagnosis of rolling bearing," Journal of xi'an jiaotong University, vol. 49, no. 5, pp. 73-81, 2015.

[27] L. Si, Z. Wang, C. Tan, and X. Liu, "A feature extraction method based on composite multi-scale permutation entropy and laplacian score for shearer cutting state recognition," Measurement, vol. 145, pp. 84-93, 2019.

[28] S. Mirjalili and A. Lewis, "The whale optimization algorithm," Advances in engineering software, vol. 95, pp. 51-67, 2016.

[29] W. Wang, H. Wang, H. Sun, and S. Rahnamayan, "Using oppositionbased learning to enhance differential evolution: A comparative study," in Proc. IEEE Congress on Evolutionary Computation, Vancouver, Canada, 2016, pp. 71-77.

[30] S. Dang, J. P. Coon, and G. Chen, "Adaptive OFDM with index modulation for two-hop relay-assisted networks," IEEE Transactions on Wireless Communications, vol. 17, no. 3, pp. 1923-1936, 2018.

[31] G. Chen, J. Jia, and Q. Han, "Study on the strategy of decreasing inertia weight in particle swarm optimization algorithm," Journal-Xian Jiaotong University, vol. 40, no. 1, p. 53, 2006.

[32] M. M. Mafarja and S. Mirjalili, "Hybrid whale optimization algorithm with simulated annealing for feature selection," Neurocomputing, vol. 260, pp. 302-312, 2017.

[33] Z. Wang, S. Dang, S. Shaham, Z. Zhang, and Z. Lv, "Basic research methodology in wireless communications: The first course for researchbased graduate students," IEEE Access, vol. 7, pp. 86678-86 696, 2019.
[34] B. Chen, Y. Yan, L. Wang, M. Chen, C. Wang, and H. Ma, "One dimension NLM denoising method based on hasudorff distance and its application in OTLC,' in Proc. IEEE APEEC, Chengdu, China, 2019, pp. $75-79$.

[35] J. J. Saucedo-Dorantes, M. Delgado-Prieto, R. A. Osornio-Rios, and R. de Jesus Romero-Troncoso, "Multifault diagnosis method applied to an electric machine based on high-dimensional feature reduction," IEEE Transactions on Industry Applications, vol. 53, no. 3, pp. 3086-3097, 2017.

[36] M. Van and H.-J. Kang, "Bearing-fault diagnosis using non-local means algorithm and empirical mode decomposition-based feature extraction and two-stage feature selection," IET Science, Measurement \& Technology, vol. 9, no. 6, pp. 671-680, 2015.

[37] Q. Yang and J. Wang, "A wavelet based multiscale weighted permutation entropy method for sensor fault feature extraction and identification," Journal of Sensors, vol. 2016, 2016.

[38] F. Xu and W. T. Peter, "A method combining refined composite multiscale fuzzy entropy with PSO-SVM for roller bearing fault diagnosis," Journal of Central South University, vol. 26, no. 9, pp. 2404-2417, 2019.

[39] M. Abdel-Basset, D. El-Shahat, I. El-henawy, V. H. C. de Albuquerque, and S. Mirjalili, "A new fusion of grey wolf optimizer algorithm with a two-phase mutation for feature selection," Expert Systems with Applications, vol. 139, p. 112824, 2020.

[40] A. Unler and A. Murat, "A discrete particle swarm optimization method for feature selection in binary classification problems," European Journal of Operational Research, vol. 206, no. 3, pp. 528-539, 2010.

[41] M. A. Elaziz, A. A. Ewees, R. A. Ibrahim, and S. Lu, "Oppositionbased moth-flame optimization improved by differential evolution for feature selection," Mathematics and Computers in Simulation, vol. 168, pp. 48-75, 2020.

[42] A. A. Ewees, M. A. Elaziz, and E. H. Houssein, "Improved grasshopper optimization algorithm using opposition-based learning," Expert Systems with Applications, vol. 112, pp. 156-172, 2018.

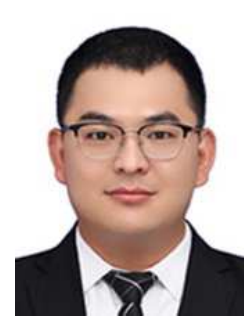

Yan Yan received the master's degree in electric engineering from Lanzhou Jiaotong University, Lanzhou, China, in 2017. He is currently pursuing the Ph.D. degree with Hohai University, Nanjing, China. His research interests include intelligent monitoring of electrical equipment, condition monitoring, and fault diagnosis of electrical equipment.

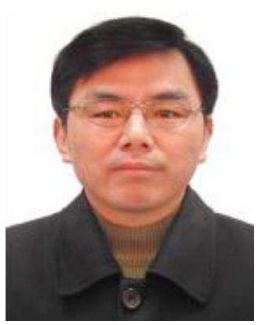

Hongzhong Ma (M'18) was born in China, in 1962. He received his B.S., M.S. and Ph.D. degrees from Southeast University, Nanjing, China, in 1989, 1994 and 2002, respectively. He is presently working as a Professor at Hohai University, Nanjing, China. His current research interests include high voltage insulation technology, condition monitoring, fault diagnosis of electrical equipment, and electrical machines and drives. 


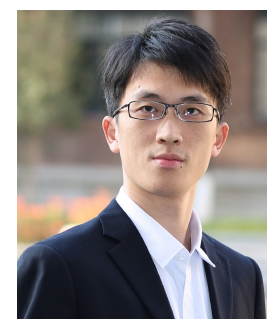

Miaowen Wen (SM'18) received the Ph.D. degree from Peking University, Beijing, China, in 2014. From 2012 to 2013, he was a Visiting Student Research Collaborator with Princeton University, Princeton, NJ, USA. He is currently an Associate Professor with South China University of Technology, Guangzhou, China, and a Hong Kong Scholar with The University of Hong Kong, Hong Kong. He has published a Springer book entitled Index Modulation for $5 \mathrm{G}$ Wireless Communications and more than 100 journal papers. His research interests include a variety of topics in the areas of wireless and molecular communications.

Dr. Wen was the recipient of four Best Paper Awards from the IEEE ITST'12, the IEEE ITSC'14, the IEEE ICNC'16, and the IEEE ICCT'19. He was recognized as an Exemplary Reviewer for the IEEE COMMUNICATIONS LETTERS in 2017 and the IEEE TRANSACTIONS ON COMMUNICATIONS in 2019. He has served on the Editorial Boards of the IEEE ACCESS, and the EURASIP Journal on Wireless Communications and Networking, and a Guest Editor for IEEE JOURNAL ON SELECTED AREAS IN COMMUNICATIONS (Special Issue on Spatial Modulation for Emerging Wireless Systems) and for IEEE JOURNAL OF SELECTED TOPICS IN SIGNAL PROCESSING (Special Issue on Index Modulation for Future Wireless Networks: A Signal Processing Perspective). Currently, he is serving as an Editor for the IEEE TRANSACTIONS ON COMMUNiCATIONS, the IEEE COMmUNiCATIONS LetTeRs, and the Physical Communication (Elsevier).

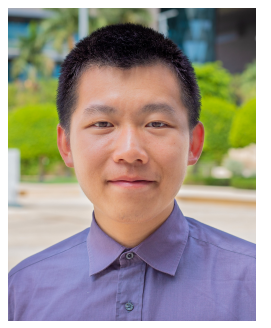

Shuping Dang (S'13-M'18) received B.Eng (Hons) in Electrical and Electronic Engineering from the University of Manchester (with first class honors) and B.Eng in Electrical Engineering and Automation from Beijing Jiaotong University in 2014 via a joint '2+2' dual-degree program. He also received D.Phil in Engineering Science from University of Oxford in 2018. Dr. Dang joined in the R\&D Center, Huanan Communication Co., Ltd. after graduating from University of Oxford and is currently working as a Postdoctoral Fellow with the Computer, Electrical and Mathematical Science and Engineering Division, King Abdullah University of Science and Technology (KAUST). His current research interests include communications science, signal processing, and optimization theory.

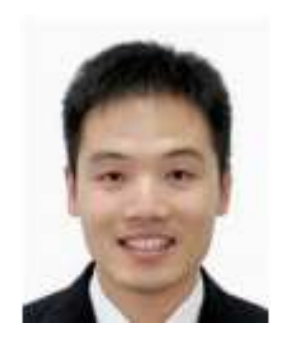

Honghua Xu received his B.S. (2004), M.S (2007) and Ph.D. (2011) degrees from the Institute of Microelectronics and Peking University, Beijing, China. Since 2011, he has been working with the Nanjing Power Supply Company, Jiangsu Electric Power Company, Nanjing, Jiangsu, China. His research interests focus on condition monitoring and fault diagnosis of power systems, smart grids, and the integration of renewable energy sources in the grid. 